\title{
Biomimetic adaptive impedance control in physical Human Robot Interaction
}

\author{
Jacopo Buzzi, Andrea Passoni, Giulio Mantoan, Maximiliano Mollura, Elena De Momi
}

\begin{abstract}
Human Robot Interaction has become a key point in the development of new robotic interfaces and controllers. In traditional control schemes for teleoperation, master devices are unaware of the user's arm dynamic characteristics, as well as of the complex motor control strategies adopted to perform the task. In this work, we propose a novel impedance controller to regulate the master device's dynamic properties based on the estimation of user's arm stiffness, with the aim of improving shared task performance. We developed a virtual planar reaching task, and we evaluated arm end-point stiffness's main axis changes in magnitude and direction using a non disruptive offline musculoskeletal model-based algorithm. Based on the stiffness modulation, the biomimetic variable impedance controller to adapt the master device's damping matrix. The direction of maximal damping was aligned with the estimated direction of maximal stiffness (Enhancing field), or to the perpendicular to the stiffness main axis (Isotropic field). The task performances under the biomimetic impedance controllers were tested and compared with the null damping condition. The results showed an increase in task performance, in terms of positional error and overshoots, with both biomimetic controllers. The analysis proved the potentiality of the biomimetic impedance modulation controller in terms of execution accuracy.
\end{abstract}

\section{INTRODUCTION}

In the last years, a growing number of applications have showcased the great potentiality of introducing robotic assistances, from manufacturing to automotive, entertainment and health-care. Depending on the application, users and robots interact, sharing mutual information about intentions and internal states. This information can be conveyed through different means of communication, comprising, among the others, visual and audio clues, gestures [1], [2], forces and torques [3]-[5].

When humans and robots physically interact (Physical Human-Robot Interaction or pHRI), two dynamically and kinematically different entities are coupled, creating a complex system. The effect of the interaction forces on the coupled system's state depend on the relation between the human's dynamic characteristics and the robot's mechanical properties. While the first are controlled by the Central Nervous System (CNS) and change with respect to muscular co-contraction and joint configuration [6], based on specific motor control strategies, robot's dynamic behavior is defined by its design and by the type of control adopted. Therefore, when analyzing a pHRI system, along with a complete control over the robot dynamics, a deep understanding of

The authors are with the Department of Electronics, Information and Bioengineering, Politecnico di Milano, Via Giuseppe Colombo 40, Milan, 20133, Italy. jacopo.buzzi@polimi.it the users motor strategies adopted during the execution of the task is of paramount importance.

Based on the nature of the physical interaction with the robot, different types of pHRI can be identified: firstly, users and robots can interact while performing a shared task. In this case, users don't hold the robot directly, but forces are exchanged while working on the same shared task i.e. during lifting and handling of heavy objects [7] or when cooperating to assemble parts within an industrial process [8].

Moreover, a rigid connection can be established between human's limbs and robots: this is the case of rehabilitative robotics, where robots are used to help neurological patients in regaining motor capabilities [9], [10].

Users can also interact with robots' end-effectors using handles or directly moving the robots' links, as in teleoperation or shared control. Among the others, in teleoperation, users interact with robots specifically designed to follow and acquire hand movements, functioning as masters to control remotely placed slaves arms [11]. In order to preserve the user's free-hand manipulative skills, master robots shouldn't, first of all, limit the natural dexterity of the arm and should allow for the most intuitive kinematic and dynamic coupling. Many studies focused on the design of master devices to improve user-master robot interaction with the final aim of achieving higher level of dexterity, larger workspaces and higher position/orientation accuracy [12]-[14]. Some authors have given emphasis to the ergonomic aspects and user's posture while teleoperating [15], [16], while others have paid attention to the master device handle design [17]. More recently, the effects on the arm kinematics and dynamics induced by different master devices architectures have been studied in order to define which are the characteristics that might effect human motor control strategies [18], [19].

Traditionally, robots are controlled using two main paradigms: position and impedance control, reflecting the control over position or interaction dynamics [20]. While in the first case the robot reaches a desired position and orientation regardless of the forces generated, in impedance control the robot-environment's force exchange is monitored and can be used to modify robots' dynamic properties, such as its compliance or damping. In order to assure teleoperation stability while implementing force reflection, high damping coefficients are usually applied to the master device, impairing the possibility of conveying small interaction forces to the user arm [21]. To overcome this limitation, adaptive impedance control was introduced [22], [23]: a time-varying position-dependent dynamic model of the environment is obtained and used to adapt the master robot impedance. When 
facing with compliant environments, which are less prone to generate high interaction forces and cause system instability, the master device's damping coefficient is reduced.

While these solutions allowed adaptations to different environments, master devices are, still, completely unaware of the continuous dynamic and kinematic changes that users undergo while performing the task. As a promising step toward the possibility of including models of the arm dynamic changes within robot control, biomimetic impedance regulation has been recently proposed [24]-[26]. In this control paradigm, dynamic characteristics of the human arm (i.e. arm stiffness) are estimated through the acquisition of bio-signals (such as muscular activations with surface electromyography signals) and are used to modify the slave robot parameters.

In other studies [27], [28], the estimated model of the arm impedance, was used to modify the robot's damping coefficient in cooperative pHRI. In these works, humans interacted with a robotic handle's whose damping coefficient was modulated to match the users arm's stiffness, evaluated using the derivative of the force exchanged by the user and the handle. These works, as well as other similar studies [29], [30], proved the potentiality of creating dynamic models of the human arm to implement controllers that improve task performance in terms of targeting accuracy and reduction of overshoots.

We present the development of a model-based stiffnessmimicking adaptive impedance controller for adjusting the dynamic properties of a master device to increase accuracy in a virtual targeting task. Using an off-line musculoskeletal model algorithm based on kinematic and dynamic data, the cartesian hand stiffness modulation was evaluated [31]. The proposed biomimetic controller accounts for the changes in the arm stiffness while performing the task, adapting the master device's damping coefficient in order to reflect the natural, anisotropic, stiffness characteristics. Through experimental acquisitions, we test our main hypothesis that the introduction of the impedance controller will increase users' performance in terms of positional error and overshoots from the targets.

\section{Materials And Methods}

In the first experiment, 7 users performed a virtual teleoperation targeting task manipulating a 7DoFs master device. Joint angles and surface EMG signals were used to obtain an offline computation of human stiffness. The estimated values of the average arm stiffness were used to define the damping coefficients adopted in the master robot variable impedance controller. In a second experiment, 12 users tested the performance of the variable impedance controller performing the same bi-dimensional targeting task.

\section{A. Task design}

In the targeting task each user interacted with the master device to move a virtual stylus shaped end effector tool on eight target points $\left(T_{1}, T_{i}, \ldots, T_{T}\right.$ with $\mathrm{T}=8$, see Fig. 1$)$. These targets lie on a plane and they are distributed to form

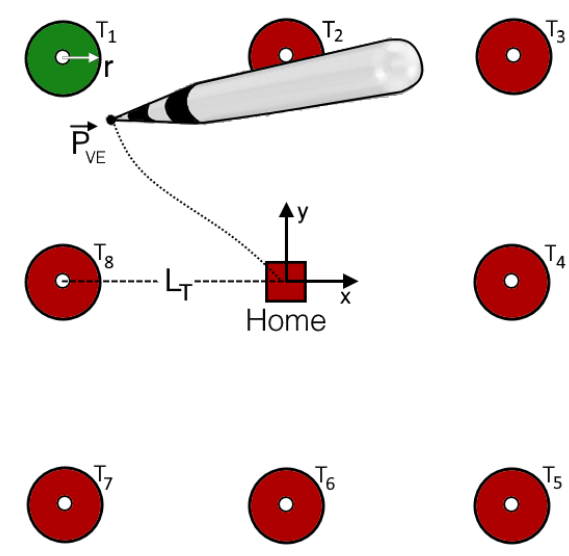

Fig. 1. Starting from the central Home, the user has to reach every single target point $T_{i}$ (arranged along the sides of a square), positioning the end effector point $\left(\mathbf{P}_{V E}\right)$ as precisely as possible in the center of the target for $1 s$ and come back to the Home. Circular targets with a radius $r=50 \mathrm{~mm}$ are are distributed on a square with semi-side $L_{T}=300 \mathrm{~mm}$. The virtual reference frame $(\mathrm{x}-\mathrm{y})$ is centered on the Home position.

a square whose semi-side length $L_{T}$ is $300 \mathrm{~mm}$. Starting from the center of the square, every user was asked to reach each target point (reaching phase) and, after having kept the position for 1s (targeting phase), to come back to the central "Home" position (see Fig. 1). During the task execution, one circle at a time changed color from red to green, guiding the user towards the correct target. Once the target's outer circle ( $r=50 \mathrm{~mm}$ ) was crossed, users had to place the tool's end effector in the inner circle (white dots in Fig. 1) as precisely as possible, while a counter measured $1 s$ before indicating users to go back to the home position and moving to the next target. The targeting phase was arbitrarily limited to a time frame of one second to create a fast paced task while still challenging users in trying to minimize the targeting error after the ballistic reaching phase.

The virtual task was developed using V-Rep (Coppelia Robotics GmbH, Zurich, Switzerland), a robot simulation program that can be interfaced through the Robotic Operating System (ROS) [32] network with the master device and with the data acquisition framework.

\section{B. Master Device}

Users performed the targeting task using the Sigma7 interface (Force Dimension, Nyon, Switzerland), a hybrid parallel/serial link master device, gravity compensated and characterized by 6 DoFs plus 1 grip control, with a resolution of $0.0015 \mathrm{~mm}$ and $0.013 \mathrm{deg}$ and an elliptical dome workspace with radiuses of approximately $190 \times 130 \mathrm{~mm}$. The task was designed to fit within the master device workspace in such a way as not to use the device clutching option which decouples the virtual tool position from the master device end effector position.

\section{Stiffness Estimation}

The offline stiffness estimation algorithm follows the steps presented in [31]. The users' arm and thorax kinematics are measured using optoelectronic cameras (Vicra - Northern 
Digital, Ontario, Canada, $20 \mathrm{~Hz}$ sampling rate) and electromagnetic sensors (Aurora - Northern Digital, Ontario, 30 $\mathrm{Hz}$ sampling rate) and 10 surface EMG signals are acquired using a multichannel ADC (TMSi Porti - Twente Medical Systems International, Oldenzaal, Nederland, $2048 \mathrm{~Hz}$ sampling rate).

Exploiting an already validated musculoskeletal model [33] developed in OpenSim (see Fig. 2), the arm joint angles variations $\vartheta$ are computed from the marker movements through inverse kinematics. Using an EMG informed inverse dynamic toolbox [34] the muscle stiffness can be estimated and projected in the joint space using the muscle Jacobian. From the joint stiffness matrix, using the geometric Jacobian, the Cartesian stiffness at the hand has been obtained. To geometrically represent the Cartesian stiffness, using the Singular Value Decomposition (SVD), the left singular vectors and values are extracted. The first singular vector represents the direction and magnitude of the stiffness main axis.

The modulation in the stiffness main axis dimension $\left(K_{\max , i}(d)\right)$ with respect to the $\mathrm{x}-\mathrm{y}$ distance $(d=$ $\left.\sqrt{\left(P_{x, V E}-T_{x, i}\right)^{2}+\left(P_{y, V E}-T_{y, i}\right)^{2}}\right)$ between the target position $\left(T_{i}\right)$ and the tool end-effector position $\left(\mathbf{P}_{V E}\right)$ during task execution are evaluated for each $i^{\text {th }}$ target. In the same way, the angle between the main stiffness axis and the $\mathrm{x}$ axis from the task reference frame $\left(\hat{\theta}_{i}\right)$ for the $i^{\text {th }}$ target is projected on the $x-y$ plane and used as indication of the stiffness ellipse's direction.

\section{Biomimetic adaptive impedance controller}

The estimated changes in the arm stiffness acquired in the initial experimental phase are used to tune the master device's damping matrix (see Fig. 2. From the initial experiment, a single average profile or arm stiffness modulation is extracted along with the 8 main axis directions corresponding to the different task targets. The stiffness changes and its directions are then used to define the variations in the master device dynamic proprieties that are tested in the second experiment.

Humans increase arm stiffness in order to achieve high precision during the execution of targeting tasks, increasing stability against internal and external noise. Following an already validated approach [28], the proposed biomimetic impedance controller increases the master device's damping coefficients to match the increase in arm stiffness.

The force generated by the master device $(\mathbf{F}(t))$ at the $t^{t h}$ time frame can be expressed as sum of elastic, damping and inertial components:

$$
\mathbf{F}(t)=\mathbf{K} \cdot \mathbf{P}_{M D}(t)+\mathbf{D} \cdot \dot{\mathbf{P}}_{M D}(t)+\mathbf{I} \cdot \ddot{\mathbf{P}}_{M D}(t)
$$

Where $\mathbf{K}$ is the elastic coefficient matrix, $\mathbf{D}$ is the robot's damping matrix, $\mathbf{I}$ is the robot's matrix of inertia (see Fig. 2), $\mathbf{P}_{M D}(t)$ and $\ddot{\mathbf{P}}_{M D}(t)$ are the Master Device (MD) endeffector position and acceleration in the MD reference frame.

The damping component of the forces generated by the adaptive impedance controller $\left(\mathbf{F}_{C}(d, t)=\mathbf{D} \cdot \dot{\mathbf{P}}_{M D}(t)\right)$ as a function of the distance from the target $d$ and the $t$ time

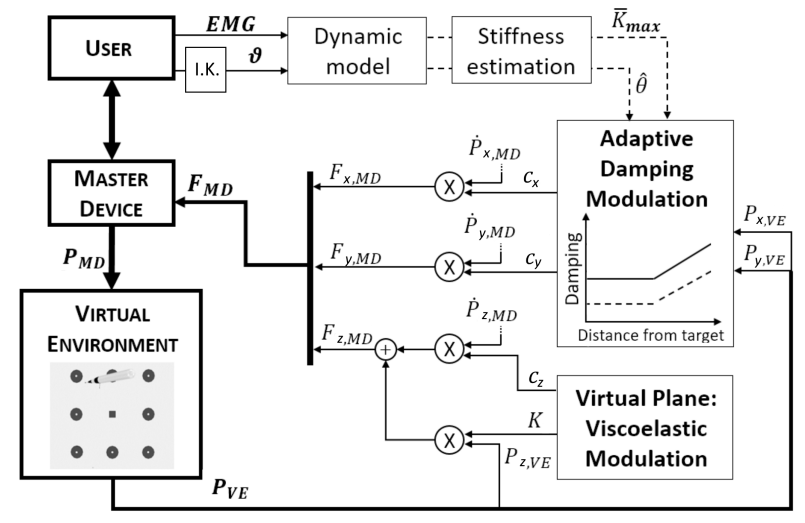

Fig. 2. Schematic of the biomimetic adaptive impedance controller: using joint angles $(\vartheta)$ and EMG signals combined with an EMG informed inverse dynamic algorithm, users' stiffness is estimated. Mean stiffness modulation $\left(\bar{K}_{\max }\right)$ and orientation ( $\hat{\theta}$, with respect to the virtual plane $\mathrm{x}$ axis) are used to generate the adaptive damping modulation $\left(c_{x}, c_{y}\right)$. The master device's speeds on the $\mathrm{x}-\mathrm{y}$ plane $\left(\dot{P}_{x, M D}, \dot{P}_{y, M D}\right)$ are used to compute the corresponding damping forces $\left(F_{x, C}, F_{y, C}\right)$. Similarly, a viscoelastic virtual plane produces forces along the $\mathrm{Z}$ axis $\left(F_{z, M D}\right)$ obtained from the sum of the elastic $\left(k_{z}\right)$ and damping ( $c_{z}$ components)

frame can be obtained as follows:

$$
\mathbf{F}_{C}(d, t)=\mathbf{C}(d) \cdot \mathbf{R}_{z}\left(\psi_{i}\right) \cdot \dot{\mathbf{P}}_{M D}(t)
$$

where $\mathbf{C}(d)$ is the damping coefficients matrix, $\dot{\mathbf{P}}_{M D}(t)$ is the MD end-effector speed and $\mathbf{R}_{z}\left(\psi_{i}\right)$ is the $3 \times 3$ rotation matrix around the $\mathrm{z}$-axis that is used to rotate the damping coefficients direction on the $x-y$ plane (see Fig. 3B).

The same equation can be also expressed as:

$$
\begin{gathered}
{\left[\begin{array}{c}
F_{x, C}(d, t) \\
F_{y, C}(d, t) \\
F_{z, C}(d, t)
\end{array}\right]=\left[\begin{array}{ccc}
c_{\min }(d) & 0 & 0 \\
0 & c_{\max }(d) & 0 \\
0 & 0 & c_{z}
\end{array}\right]} \\
{\left[\begin{array}{ccc}
\cos \left(\psi_{i}\right) & -\sin \left(\psi_{i}\right) & 0 \\
\sin \left(\psi_{i}\right) & \cos \left(\psi_{i}\right) & 0 \\
0 & 0 & 1
\end{array}\right] \cdot\left[\begin{array}{l}
\dot{P}_{x, M D}(t) \\
\dot{P}_{y, M D}(t) \\
\dot{P}_{z, M D}(t)
\end{array}\right]}
\end{gathered}
$$

where $c_{\min }(d)$ and $c_{\max }(d)$ represent the adaptation of the module of the two axes of damping (along $x$ and $y$ ) with respect to changes in the target-tool distance $d$. In order to reflect arm stiffness' anisotropy, we generated a non-isotropic elliptical damping field, whose axis dimensions ( $c_{\min }$ and $c_{\max }$ ) are tuned to match the changes in the mean arm stiffness module $\bar{K}_{\max }$ when approaching targets. The linear increase in the mean arm stiffness module $\bar{K}_{\max }$ is matched with a linear increase in the bi-dimensional task space virtual viscosity. On the other hand, $c_{z}$ represents a constant damping coefficient that we used to avoid possible instability caused by the elastic virtual plane (see II-E).

At $d^{\text {th }}$ distance from the target, $c_{\min }$ and $c_{\max }$ are defined as follows:

$$
c_{\min }(d)= \begin{cases}c_{\min , 1} & d \geq L_{T} / 2 \\ c_{\min , 0}-\frac{\left(c_{m i n, 0}-c_{\min , 1}\right) \cdot d(t)}{L_{T} / 2} & d<L_{T} / 2\end{cases}
$$




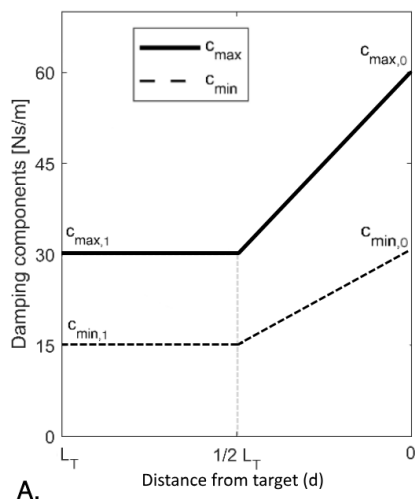

A.

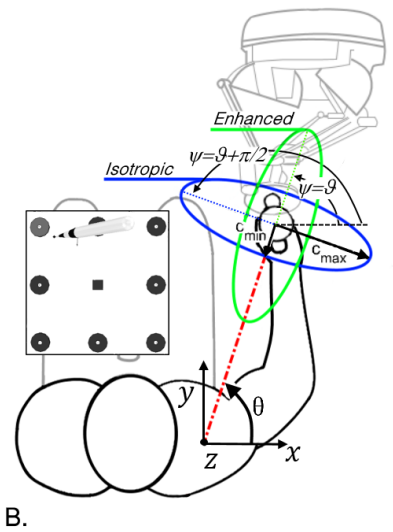

Fig. 3. A. $c_{\max }$ and $c_{\min }$ as function of $d$. B. Subject posture during task execution. The main axis of the stiffness ellipse (red dash-dot line), corresponds to the shoulder-hand direction. In green: the Enhancing damping coefficient field, in blue, the Isotropic one.

$$
c_{\max }(d)= \begin{cases}c_{\max , 1} & d \geq L_{T} / 2 \\ c_{\max , 0}-\frac{\left(c_{\max , 0}-c_{\max , 1}\right) \cdot d(t)}{L_{T} / 2} & d<L_{T} / 2\end{cases}
$$

Where $c_{\min , 0}$ and $c_{\max , 0}$ are respectively the maximal and minimal values of $c$ (when $d=0$ ) and $c_{\min , 1}$ and $c_{\max , 1}$ are the minimal and maximal values of $c$ (when $d=L_{T}$ ).

The maximal damping variation $\left(\Delta c_{\max }=c_{\max , 0}-\right.$ $\left.c_{\max , 1}\right)$ was obtained from the estimated variation of maximal stiffness $\Delta K_{\max }=K_{\max , 0}-K_{\max , 1}$ as follows:

$$
\Delta c_{\max }=\Delta K_{\max } \cdot s_{\max }
$$

Where $s_{\max }=\frac{3}{2} \frac{s}{m^{2}}$ is the scaling coefficient that converts the arm stiffness modulation into the master device's damping modulation. In the same way, for $\Delta c_{\min }$, a scaling factor $s_{\min }=\frac{3}{4} \frac{s}{m^{2}}$ was used. The scaling factors $\left(s_{\max }\right.$ and $\left.s_{\min }\right)$ were experimentally defined to occupy the maximal range of forces that the master device was capable of generating while maintaining a reliable force feedback within the task's workspace. In order to test different conditions, expert and naive users were asked to repeatedly perform the targeting task at different speed and with different scaling factors.

The resulting variation of maximum and minimum components of the damping coefficient as a function of $d$ is shown in Fig. 3.A.

Two different damping fields were generated:

1) Enhancing damping field: In the first case, the maximal damping coefficient is directed along the axis of maximal stiffness. This can be achieved imposing $\psi=\bar{\theta}_{i}$ in Eq. 3 , therefore applying rotations around the $\mathrm{z}$ axis equal to the displacement between the main axis of stiffness and the $x$ axis $\left(\bar{\theta}_{i}\right)$ for each of the eight targets (see Fig. 3.B, green ellipse). The effects of this damping field enhance the natural stiffness directionality, increasing the effect of the stiffness ellipse anisotropy.

2) Isotropic damping field: In the second case, the maximal damping coefficient is directed along the perpendicular to the stiffness main axis, imposing $\psi=\bar{\theta}_{i}+\pi / 2$ (see Fig. 3.B, blue ellipse). In this case, we aim at obtaining a more isotropic workspace for the user hand by summing the effects of the natural impedance control with the controller generated damping forces.

\section{E. Visco-elastic Plane}

Perpendicularly to the virtual plane of the task $(x-y)$, a viscoelastic force is applied in order to help the user to steady the tool-tip $\mathrm{P}_{z, M D}$ on the $\mathrm{z}$ dimension. This solution was introduced to compensate for the lack of $3 \mathrm{D}$ perception that users suffered while performing the tasks on a flat bidimensional screen.

\section{F. Experimental protocol}

Since an off-line method for the estimation of the arm end-point stiffness was adopted, the biomimetic adaptive impedance controller's effects were evaluated on a successive experimental session.

1) Arm stiffness estimation: For the stiffness characterization, we recruited 7 healthy subjects ( 4 female and 3 male, mean age $23 \pm 1.5$ y.o) Each user was seated in a comfortable chair in front of a monitor and, after having been instructed about the task he/she was going to perform, he/her was asked to execute 10 trials while trying to maintain the hand movement on a constant plane. The monitor was put as flat as possible to make it parallel to the pane in which the task was performed.

2) Variable impedance controller: For testing the performance of the stiffness-based variable impedance controller, we recruited 12 right-handed subjects (4 females and 8 males, mean age $24 \pm 2.8$ y.o.) who had no experience with the master controller. Each subject performed 10 trials of the same virtual task under 3 different force conditions (30 overall repetitions): no impedance regulation ( Null), Enhancing damping field and Isotropic damping field. The execution order was randomized, assuring to have the three different conditions tested an equal overall number of times.

All the involved subjects provided informed written consent, in accordance with the recommendations of Politecnico di Milano Ethical committee Board. All subjects gave written informed consent in accordance with the Declaration of Helsinki.

\section{G. Performance Indexes}

To assess each subject's performance during task execution, the following indexes were evaluated for each damping field condition:

- Maximal Error $\left(E_{\max , i}\right)$ : Maximal distance from the $i^{t h}$ target during the targeting phase.

$$
E_{\max , i}=\max _{\left[t_{c}: t_{o f f}\right]} d(t)
$$

Where $\mathrm{t}_{c}$ and $\mathrm{t}_{\text {off }}$ are, respectively, the time frames when the targeting phase starts and ends.

- Integral Error $\left(E_{\text {int }, i}\right)$ : Error computed as integral of the distance from the $i^{\text {th }}$ target normalized by the duration of the resting phase.

$$
E_{i n t, i}=\frac{\int_{t_{c}}^{t_{o f f}} d(t) d t}{t_{o f f}-t_{c}}
$$


- Unacceptable Error $\left(E_{u, i}\right)$ : Error computed as for $\mathrm{E}_{i n t, i}$, but considering only the distances from the $i^{\text {th }}$ target higher than the radius of the target $r$.

$$
E_{u, i}=\frac{\int_{t_{c}}^{t_{o f f}} d(t) d t}{t_{o f f}-t_{c}} \quad d(t)>r
$$

- Overshoots $(O)$ : Number of times that the subjects went out from the $i^{t h}$ target during the resting phase.

- Normalized Traveled Length (NTL): Normalized length of the path traveled by the subjects during each reaching phase; it is expressed as:

$$
N T L_{i}=1-\frac{\sum_{k=1}^{N} \sqrt{\Delta P_{x, V E, i}^{2}+\Delta P_{y, V E, i}^{2}}}{L_{T}}
$$

Where $\mathrm{N}$ is the number of sampled positions of the virtual tool tip during the reaching phase, $\Delta P_{x, V E, i}$ and $\Delta P_{y, V E, i}$ represent the variation in the $\mathrm{x}$ and $\mathrm{y}$ components of the tool's end-effector position in the virtual environment reference frame (VE) with respect to the previous $(k-1)^{\text {th }}$ sample.

- Reaching Time $\left(t_{R, i}\right)$ : Time needed to reach the $i^{t h}$ target from the center position.

$$
t_{R, i}=t_{c}-t_{o n}
$$

Where $\mathrm{t}_{\text {on }}$ and $\mathrm{t}_{c}$ are, respectively, the time frames when the reaching phase and the targeting phase starts.

\section{H. Results analysis}

1) Stiffness Estimation: The reaching and targeting phases of the task were extracted for each subject, repetition and $i^{\text {th }}$ target. The stiffness ellipsoid's main axis dimension $K_{\max }(d)$ and direction $\hat{\theta}(d)$ were extracted and, for each subject, their distribution normality between different repetitions was evaluated (Lilliefors test, $\alpha=0.05$ ). Since the data, for all the subjects, was normally distributed, the mean stiffness main axis dimension for each target throughout the normalized time was obtained $\bar{K}_{\max , i}(d)$ as well as the mean direction of stiffness for each target $\bar{\theta}_{i}(d)$.

Since the same stiffness modulation was observed for all the targets, a single modulation $\bar{K}_{\max }(d)$ was obtained from the mean through the eight targets directions. Linear regression was used to describe the $\bar{K}_{\max }(d)$ modulation. No significant changes in the $\bar{\theta}_{i}(d)$ were seen throughout the task progression, with the data appearing normally distributed (Lilliefors test, $\alpha=0.05$ ), therefore, a single mean value of $\bar{\theta}_{i}$ was extracted for each target.

2) Variable impedance control performances: The first experimental session, for each subject, regardless of the force condition (Null, Enhancing or Isotropic) was considered as a training phase during which users had time to familiarize with the master device and with the task. For this reason, the first experimental session was excluded from the analysis.

For each index, within the same subject and force condition, the data distribution through the 10 repetitions was evaluated (one-way ANOVA, $\alpha=0.05$ ). Since in some cases the first repetition of each force condition was found to be significantly different from the others (Tukey's range test $\alpha=0.05)$ nine task repetitions $(2: 10)$ were considered for the analysis.

For each user and each of the eight targets, the statistical distribution for the nine considered repetitions of the aforementioned indexes was evaluated using a normality test (Lilliefors $\alpha=0.05$ ). Since the data distribution was normal, and no significant differences were found between different targets, the mean value for each subject and force was extracted. The differences between the three force conditions for each index were evaluated using a one-way ANOVA test ( $\alpha=0.05$ ), followed by post-hoc analysis (Tukey's honestly significant difference procedure $\alpha=0.05$ ).

All the inferential statistic analysis were conducted with the Statistics and Machine Learning Toolbox for Matlab 2017b (Mathworks, Natick, Massachusetts, US)

\section{RESULTS}

\section{A. Stiffness Estimation}

The mean stiffness modulation profile $\bar{K}_{\max }(d)$ with respect to $d$, as well as the first and third quartiles, are shown in Fig 4. While in the first part of the movement, (from 0 to $\left.\frac{1}{2} L_{T}\right) \bar{K}_{\max }$ is constant in both mean and interquartile range, an ascending trend can be observed in the second half of the movement and a linear regression approximates this part of task ( $\rho=0.887, p=0.0003$ see red line in Fig. 4).

The stiffness ellipse orientations were found to be normally distributed (Lilliefors test, $\alpha=0.05$ ), therefore, the mean orientation was extracted for each target. $\bar{\theta}_{i}$ (angle between the stiffness main axis and the $\mathrm{x}$ axis in the task's reference frame) ranges between 50 and $60 \mathrm{deg}$ (see Fig. 4.B), grossly overlapping with the shoulder-hand direction.

\section{B. Variable impedance control performances}

Figure 5 shows the inter subjects variability for each index's mean value. The Null damping condition shows worst results in terms of Maximal Error $\left(E_{\max }\right)$ with respect to the Enhancing $(p=0.006)$ and the Isotropic $(p=0.026)$ damping fields. Under the Null damping condition users showed a median $E_{\text {max }}$ of $2.78 \mathrm{~mm}$ while targeting the center of the $30 \mathrm{~mm}$ circular target. While performing the task with the biomimetic variable impedance controllers, this error was reduced to less than $2.35 \mathrm{~mm}$ (corresponding to a $15.5 \%$ increase in targeting accuracy).

Similar results can be seen in the Integral Error $\left(E_{\text {int }}\right)$ ( $p=0.022$ with respect to Enhancing and $p=0.045$ with respect to Isotropic damping field) and in the Unacceptable Error $\left(E_{u}\right)(p=0.004$ with respect to Enhancing and $p=$ 0.024 with respect to Isotropic damping field). For the $E_{u}$, the Null condition is characterized by a larger interquartile distance. A very similar trend is seen in the Overshoots index (O), where the Null damping condition shows significant worse performance with respect to the Enhancing ( $p=0.013$ ) and Isotropic $(p=0.043)$ conditions and larger interquartile distance. While users overshot targets around one time under the effects of the biomimetic variable damping controllers, 


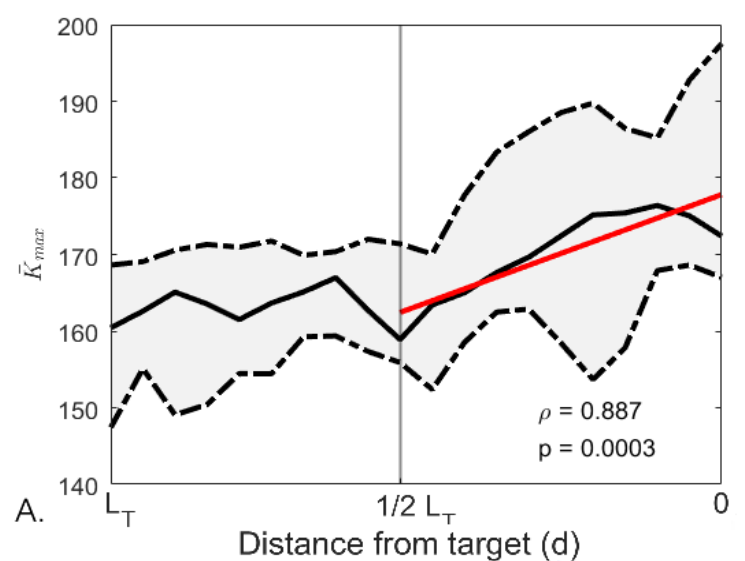

B.

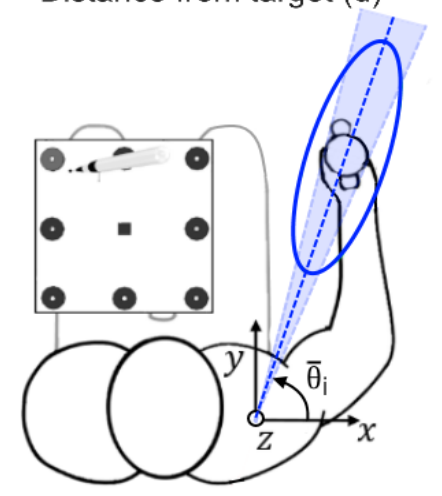

Fig. 4. Mean stiffness modulation profile and stiffness ellipse orientation. A. the solid black line represents the mean stiffness $\bar{K}_{\max }(\mathrm{d})$ over the different targets, while the dashed black lines represent the first and third quantiles. The solid red line shows the linear regression, whose significance $(p)$ and $\rho$ values are also reported. B. The main axis of the mean stiffness ellipse is represented with a dashed line along the shoulder-hand direction. $\bar{\theta}$ is the angle between the task's $\mathrm{x}$-axis and the direction of maximal stiffness. The shaded area represents $\bar{\theta}$ variability around the mean

when no damping was applied users surpassed the target around six times during task repetitions.

On the other hand, no differences were found in terms of Normalized Tool movement Length (NTL) and target reaching time $\left(t_{R}\right)$ although, for the Isotropic damping condition, the NTL interquartile range is significantly smaller.

No significant differences between the Enhancing and Isometric damping fields were found. A very small, non significant trend can be seen in $E_{\max }, E_{u}$ and for the Overshoots, where the median and mean index values for the Isotropic damping field are slightly higher with respect to the Enhancing condition. On the contrary, for the reaching time $t_{r}$, the minimal mean value was achieved under the Isotropic force condition.

\section{DISCUSSION}

The arm stiffness modulation was evaluated in terms of maximal value and main axis direction during the execution of a teleoperated targeting tasks. The results showed an increase in the arm stiffness when approaching the targets, while a rather constant main axis direction was maintained through the different targets. From the results it can be inferred that in the first part of the reaching task the maximal stiffness exerted by the users is almost constant: users are still relatively far from the target, producing a fast movement rather than a precise one. While approaching the target, maximal stiffness increases, reflecting, as expected, the necessity of increasing the hand stability against internal and external noise, to achieve greater precision.

Regarding the stiffness main axis direction, the results showed no significant differences in the ellipses mean orientation among different targets because of the very slight changes in the joint configuration of the arm required by the task in reaching the different target points. The direction along which subjects exerted the maximal stiffness modulation was the shoulder-hand one in accordance with previous studies [35]-[38].

From this modulation, a biomimetic variable impedance controller for the master device was developed and tested. We chose to modulate the master robot's damping, rather than its stiffness, to maintain a seamless and safe humanrobot interaction between users and the master device. In fact, while changes in the robot's stiffness have been used to simulate different types of constraints and feedbacks [39], these controllers have to observe strict rules in order to assure system's stability. Changes in the robot's damping, instead, are less disruptive of the users hand dynamics therefore allowing for improved transparency [28]. The results showed that the biomimetic impedance modulation increased users targeting performances. The damping positively influenced users performance, both in terms of maximal displacement from the task goal, as well as in their capability of finely finding the center of the targets' circle during the given time.

The mean number of overshoots per experiment shows that the variable impedance control allows users to perform significantly better while introducing no significant differences in terms of end effector's traveled length nor reaching time: users were therefore facilitated in finding an advantageous kinematic and dynamic matching with the master device.

The results obtained are in accordance with previous experiments: in [30], Beretta et al., showed that the introduction of a variable impedance controller increased expert and naive users performance during a human-robot cooperative point targeting task. In the aforementioned analysis, a robotic arm damping matrix was modulated isotropically based on an a priori knowledge of the task performed, which requested an increased precision in its final linefollowing part. Coherently, a similar study from Duchaine and Gosselin showed how the introduction of an arm stiffness based adaptation of a robotic handle damping factor led to significant improvements in terms of overshoots reduction in a cooperative drawing task [28].

No significant differences between the Enhancing and Isotropic force conditions was found: users behaved similarly when facing an augmentation of the natural arm stiffness (achieved when the maximal damping was aligned with the direction of maximal stiffness) and when the same damping was directed along the perpendicular to the stiffness main axis. This results may be interpreted in different ways: first of all it is possible that the difference between the damping 

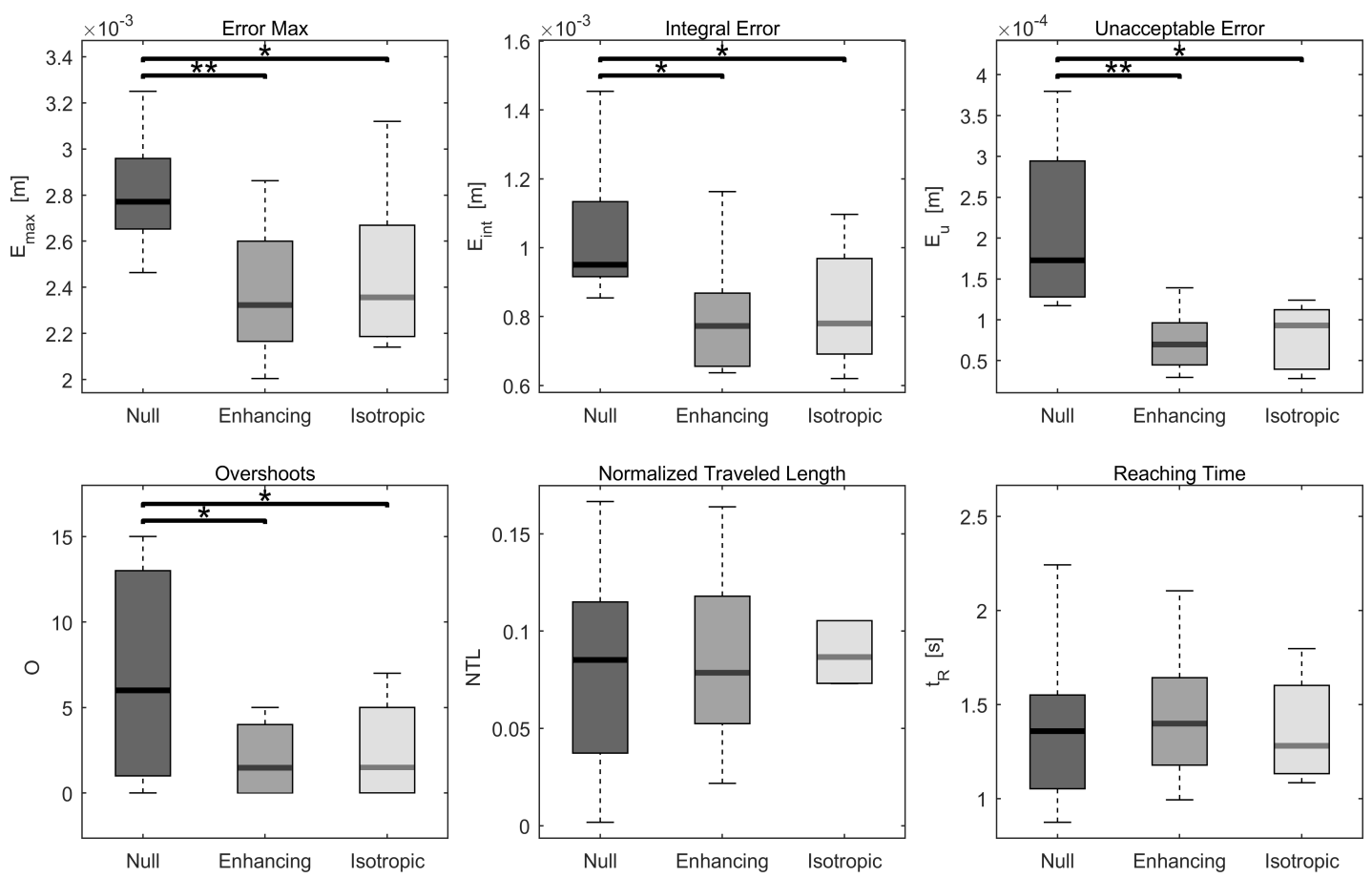

Fig. 5. Statistical distributions for the three force conditions throughout the six indexes analyzed. Statistical significance (evaluated with one way-ANOVAs and post-hoc tests) is indicated with lines and stars (* for $p<0.05$, ** for $p<0.01$ )

coefficient's magnitude in the two directions is too small to introduce significant effects in the arm and hand dynamics. It is, therefore, possible that higher damping coefficients would lead to different results. Another possible reason may be found in the capability of the human arm to cope with different robot's dynamics while achieving similar results: with both force fields, users seemed to benefit from the overall increase in damping regardless of its anisotropy and orientation.

A limitation of the present work can be found in the absence of a real-time estimation of the arm stiffness during the execution of the task. In fact, due to the offline nature of the arm's stiffness estimation adopted, two separated subsequent experiments had to be conducted, involving different subjects. This solution implies the necessity of approximating the diverse dynamic modulations that different users might exhibit to a common trend, which defines the changes in the damping coefficients presented. The adoption of an online stiffness estimation method (as in [40]), could, instead, preserve and take into consideration subjects' dynamic variability, possibly magnifying the effects of the biomimetic impedance controllers.

Another limitation can be found in the nature of the targeting task tested. In fact, while stiffness control plays an important role in the regulation of task accuracy, it is also fundamental during dynamic interactions with unstable or noisy environments. In order to fully understand the capabilities of the biomimetic impedance controller proposed, it would, therefore, be useful to test its performance under adverse conditions, for example, applying random disturbances to the users hands.

\section{CONCLUSIONS}

In the present work we developed and tested two implementations of an adaptive impedance controller in HRI during the execution of a virtual planar targeting task. The arm stiffness modulation main axis dimension and direction were evaluated through a musculoskeletal model approach based on acquired kinematic and dynamic data. In a followup experiment, the stiffness modulation was matched with corresponding changes in the master device's impedance parameters, creating two damping fields: one directed along the estimated stiffness main axis, the other perpendicular to it. In accordance with previous literature, the results showed that users benefited from the variable impedance controller in terms of positional error and overshoots but no significant difference was found between its two implementations.

Regarding the possibility of finding differences between the two biomimetic impedance regulation strategies presented, the results suggest that further analysis should be conducted, involving higher values of damping coefficients, increasing the damping fields eccentricity or studying more complex tasks.

\section{ACKNOWLEDGMENTS}

This project has received funding from the European Union's Horizon 2020 research and innovation programme under grant agreement No. H2020-ICT-2016-732515 


\section{REFERENCES}

[1] V. Richter, B. Carlmeyer, F. Lier, S. Meyer zu Borgsen, D. Schlangen, F. Kummert, S. Wachsmuth, and B. Wrede, "Are you talking to me?: Improving the robustness of dialogue systems in a multi party hri scenario by incorporating gaze direction and lip movement of attendees," in Proceedings of the Fourth International Conference on Human Agent Interaction. ACM, 2016, pp. 43-50.

[2] M. Hasanuzzaman, T. Zhang, V. Ampornaramveth, H. Gotoda, Y. Shirai, and H. Ueno, "Adaptive visual gesture recognition for human-robot interaction using a knowledge-based software platform," Robotics and Autonomous Systems, vol. 55, no. 8, pp. 643-657, 2007.

[3] J. Moll and E.-L. Sallnäs, "Communicative functions of haptic feedback," in International Conference on Haptic and Audio Interaction Design. Springer, 2009, pp. 1-10.

[4] B. D. Argall and A. G. Billard, "A survey of tactile human-robot interactions," Robotics and autonomous systems, vol. 58, no. 10, pp. 1159-1176, 2010.

[5] D. Silvera-Tawil, D. Rye, and M. Velonaki, "Artificial skin and tactile sensing for socially interactive robots: A review," Robotics and Autonomous Systems, vol. 63, pp. 230-243, 2015.

[6] A. De Santis, B. Siciliano, A. De Luca, and A. Bicchi, "An atlas of physical human-robot interaction," Mechanism and Machine Theory, vol. 43, no. 3, pp. 253-270, 2008.

[7] S. M. Rahman and R. Ikeura, "Weight-prediction-based predictive optimal position and force controls of a power assist robotic system for object manipulation," IEEE Transactions on Industrial Electronics, vol. 63, no. 9, pp. 5964-5975, 2016.

[8] P. Tsarouchi, A.-S. Matthaiakis, S. Makris, and G. Chryssolouris, "On a human-robot collaboration in an assembly cell," International Journal of Computer Integrated Manufacturing, vol. 30, no. 6, pp. 580-589, 2017.

[9] S. Bai and S. Christensen, "Biomechanical hri modeling and mechatronic design of exoskeletons for assistive applications," in Human Modelling for Bio-Inspired Robotics. Elsevier, 2016, pp. 251-272.

[10] S. Shamsuddin, "Development of human-robot interaction (hri) methodology for autism rehabilitation using humanoid robot with a telerehabilitation platform," Ph.D. dissertation, Universiti Teknologi MARA, 2015.

[11] G. Adamides, G. Christou, C. Katsanos, M. Xenos, and T. Hadzilacos, "Usability guidelines for the design of robot teleoperation: A taxonomy," IEEE Transactions on Human-Machine Systems, vol. 45, no. 2, pp. 256-262, 2015.

[12] P. Fischer, R. Daniel, and K. V. Siva, "Specification and design of input devices for teleoperation," in Robotics and Automation, 1990. Proceedings., 1990 IEEE International Conference on. IEEE, 1990, pp. $540-545$.

[13] A. Bejczy and B. Hannaford, "Man-machine interaction in space telerobotics," in Proceedings of the International Symposium of Teleoperation and control, vol. 1988, 1988.

[14] T. Burgess, D. Kuban, W. Hankins, and R. Mixon, "Operational experience and design recommendations for teleoperated flight hardware," 1988.

[15] E. Lee, A. Rafiq, R. Merrell, R. Ackerman, and J. Dennerlein, "Ergonomics and human factors in endoscopic surgery: a comparison of manual vs telerobotic simulation systems," Surgical endoscopy and other interventional techniques, vol. 19, no. 8, pp. 1064-1070, 2005.

[16] E. H. Lawson, M. J. Curet, B. R. Sanchez, R. Schuster, and R. Berguer, "Postural ergonomics during robotic and laparoscopic gastric bypass surgery: a pilot project," Journal of robotic surgery, vol. 1, no. 1, pp. 61-67, 2007.

[17] L. Santos-Carreras, M. Hagen, R. Gassert, and H. Bleuler, "Survey on surgical instrument handle design: ergonomics and acceptance," Surgical innovation, vol. 19, no. 1, pp. 50-59, 2012.

[18] J. Buzzi, C. Gatti, G. Ferrigno, and E. De Momi, "Analysis of joint and hand impedance during teleoperation and free-hand task execution," IEEE Robotics and Automation Letters, vol. 2, no. 3, pp. 1733-1739, 2017.

[19] J. Buzzi, E. D. Momi, and I. Nisky, "An uncontrolled manifold analysis of arm joint variability in virtual planar position and orientation telemanipulation," IEEE Transactions on Biomedical Engineering, pp. 1$1,2018$.

[20] S. Chiaverini and L. Sciavicco, "The parallel approach to force/position control of robotic manipulators," IEEE Transactions on Robotics and Automation, vol. 9, no. 4, pp. 361-373, 1993.
[21] N. Enayati, E. De Momi, and G. Ferrigno, "Haptics in robot-assisted surgery: challenges and benefits," IEEE reviews in biomedical engineering, vol. 9, pp. 49-65, 2016.

[22] D. A. Lawrence, "Stability and transparency in bilateral teleoperation," IEEE transactions on robotics and automation, vol. 9, no. 5, pp. 624637, 1993.

[23] L. J. Love and W. J. Book, "Force reflecting teleoperation with adaptive impedance control," IEEE Transactions on Systems, Man, and Cybernetics, Part B (Cybernetics), vol. 34, no. 1, pp. 159-165, 2004.

[24] A. Ajoudani, N. G. Tsagarakis, and A. Bicchi, "Tele-impedance based teleoperation: Peg-in-hole experimental results," in Robotics and Biomimetics (ROBIO), 2011 IEEE International Conference on. IEEE, 2011, pp. 2239-2240.

[25] A. Ajoudani, N. Tsagarakis, and A. Bicchi, "Tele-impedance: Teleoperation with impedance regulation using a body-machine interface," The International Journal of Robotics Research, vol. 31, no. 13, pp. 1642-1656, 2012.

[26] B. Huang, Z. Li, X. Wu, A. Ajoudani, A. Bicchi, and J. Liu, "Coordination control of a dual-arm exoskeleton robot using human impedance transfer skills," IEEE Transactions on Systems, Man, and Cybernetics: Systems, 2017.

[27] T. Tsumugiwa, R. Yokogawa, and K. Hara, "Variable impedance control based on estimation of human arm stiffness for human-robot cooperative calligraphic task," in Robotics and Automation, 2002. Proceedings. ICRA'O2. IEEE International Conference on, vol. 1. IEEE, 2002, pp. 644-650.

[28] V. Duchaine and C. Gosselin, "Safe, stable and intuitive control for physical human-robot interaction," in Robotics and Automation, 2009. ICRA'09. IEEE International Conference on. IEEE, 2009, pp. 33833388.

[29] N. Jarrassé, J. Paik, V. Pasqui, and G. Morel, "How can human motion prediction increase transparency?" in Robotics and Automation, 2008. ICRA 2008. IEEE International Conference on. IEEE, 2008, pp. 2134-2139.

[30] E. Beretta, E. De Momi, F. R. y Baena, and G. Ferrigno, "Adaptive hands-on control for reaching and targeting tasks in surgery," International Journal of Advanced Robotic Systems, vol. 12, no. 5, p. 50, 2015.

[31] J. Buzzi, J. Jansma, G. Ferrigno, and E. De Momi, "On the value of estimating human arm stiffness during virtual teleoperation with robotic manipulators," Frontiers in Neuroscience, vol. 11, p. 528, 2017.

[32] M. Quigley, K. Conley, B. Gerkey, J. Faust, T. Foote, J. Leibs, R. Wheeler, and A. Y. Ng, "Ros: an open-source robot operating system," in ICRA workshop on open source software, vol. 3, no. 3.2. Kobe, Japan, 2009, p. 5.

[33] K. R. Holzbaur, W. M. Murray, and S. L. Delp, "A model of the upper extremity for simulating musculoskeletal surgery and analyzing neuromuscular control," Annals of biomedical engineering, vol. 33, no. 6, pp. 829-840, 2005.

[34] C. Pizzolato, D. G. Lloyd, M. Sartori, E. Ceseracciu, T. F. Besier, B. J. Fregly, and M. Reggiani, "CEINMS: A toolbox to investigate the influence of different neural control solutions on the prediction of muscle excitation and joint moments during dynamic motor tasks," Journal of Biomechanics, vol. 48, no. 14, pp. 3929-3936, 2015.

[35] F. A. Mussa-Ivaldi, N. Hogan, and E. Bizzi, "Neural, mechanical, and geometric factors subserving arm posture in humans," Journal of Neuroscience, vol. 5, no. 10, pp. 2732-2743, 1985.

[36] T. Flash and F. Mussa-Ivaldi, "Human arm stiffness characteristics during the maintenance of posture," Experimental Brain Research, vol. 82, no. 2, pp. 315-326, 1990.

[37] J. M. Dolan, M. B. Friedman, and M. L. Nagurka, "Dynamic and loaded impedance components in the maintenance of human arm posture," IEEE Transactions on Systems, Man, and Cybernetics, vol. 23, no. 3, pp. 698-709, 1993.

[38] H. Gomi and M. Kawato, "Human arm stiffness and equilibriumpoint trajectory during multi-joint movement," Biological cybernetics, vol. 76, no. 3, pp. 163-171, 1997.

[39] N. Enayati, E. De Momi, and G. Ferrigno, "Haptics in robot-assisted surgery: Challenges and benefits," 2016.

[40] A. Ajoudani, C. Fang, N. G. Tsagarakis, and A. Bicchi, "A reducedcomplexity description of arm endpoint stiffness with applications to teleimpedance control," in Intelligent Robots and Systems (IROS), 2015 IEEE/RSJ International Conference on. IEEE, 2015, pp. 10171023. 